\title{
Per Second
}

National Cancer Institute

\section{Source}

National Cancer Institute. Per Second. NCI Thesaurus. Code C66965.

The number of occurrences of something within a second of time. 